\title{
WEAKLY STARLIKE MEROMORPHIC UNIVALENT FUNCTIONS. II
}

\section{ALBERT E. LIVINGSTON}

\begin{abstract}
A function $f(z)$ is said to be meromorphic weakly starlike if it has the form $f(z)=-\rho z F(Z) /(z-\rho)(1-\rho z)$ for $0<\rho<1$ where $F(z)$ is a member of $\Sigma^{*}$, the class of meromorphic, normalized starlike univalent functions. The coefficients of the power series expansion in $|z|<\rho$ of a meromorphic weakly starlike function are studied. The integral means of such functions are also discussed.
\end{abstract}

1. Introduction. Let $f(z)$ be meromorphic in the unit disk $\Delta=\{z|| z \mid<1\}$ with a simple pole at $z=\rho, 0<\rho<1$, and otherwise regular in $\Delta$. $f(z)$ is said to be a member of $\Lambda(\rho)$ if and only if $f(0)=1$ and there is a number $\rho_{1}$, $\rho<\rho_{1}<1$, such that with $z=r e^{i \theta}$

$$
\operatorname{Re}\left[z f^{\prime}(z) / f(z)\right]<0
$$

and

$$
\frac{1}{2 \pi} \int_{0}^{2 \pi} \operatorname{Re}\left[\frac{z f^{\prime}(z)}{f(z)}\right] d \theta=-1
$$

for $\rho_{1}<r<1$. Also let $\Lambda^{*}(\rho), 0<\rho<1$, be the class of functions $f(z)$ which have the representation

$$
f(z)=-\rho z g(z) /(z-\rho)(1-\rho z)
$$

where $g(z)=z^{-1}+a_{0}+a_{1} z+\ldots$ is a member of $\Sigma^{*}$, the class of meromorphic, normalized, starlike univalent functions [1].

The classes $\Lambda(\rho)$ and $\Lambda^{*}(\rho)$ have recently been studied by Libera and the author [8]. Functions in $\Lambda^{*}(\rho)$ are called weakly starlike meromorphic univalent functions since they are reciprocals of weakly starlike regular univalent functions introduced by Hummel [5], [6]. It was pointed out in [8] that functions in $\Lambda^{*}(\rho)$ are univalent and for each $\rho, \Lambda(\rho)$ is a subset of $\Lambda^{*}(\rho)$. Furthermore if $\rho>1 / 2, \Lambda(\rho)$ is a proper subset of $\Lambda^{*}(\rho)$, but if

$$
0<\rho<(3-2 \sqrt{2})^{1 / 2} \approx \cdot 4,
$$

then $\Lambda(\rho)=\Lambda^{*}(\rho)$.

If $f(z)$ is in $\Lambda^{*}(\rho)$ then it has a Taylor series expansion in $|z|<\rho$ of the

Received by the editors April 12, 1976.

AMS (MOS) subject classifications (1970). Primary 30A32, 30A34.

(c) American Mathematical Society 1977 
form $f(z)=1+\sum_{n=1}^{\infty} a_{n} z^{n} . \S 2$ of this paper concerns itself with the coefficients $a_{n}$ of this expansion. In particular, if $f(z)$ is real for real values of $z$, we obtain precise upper and lower bounds on $a_{n}$ for all $n$. $\$ 3$ deals with integral means of a function in $\Lambda^{*}(\rho)$.

2. Coefficient bounds. Goodman [3] studied the class $T M$ of functions $f(z)$ meromorphic in $\Delta$ with Taylor series in a neighborhood of the origin of the form $f(z)=z+\sum_{n=1}^{\infty} b_{n} z^{n}$ and which except at the poles satisfy the condition $\operatorname{Im} f(z) \operatorname{Im} z \geqslant 0$. Such functions are said to be meromorphic and typically real. Letting $T M(\rho)$ denote the subclass of $T M$ of functions for which the poles $\rho_{j}$ satisfy $\left|\rho_{j}\right| \geqslant \rho, j=1,2,3, \ldots$, Goodman proved [3] that for all $n$

$$
\left|b_{n}\right| \leqslant\left(1-\rho^{2 n}\right) / \rho^{n-1}\left(1-\rho^{2}\right) .
$$

If we assume that $f(z)$ in $\Lambda^{*}(\rho)$ is real for real values of $z$ and has the Taylor series expansion $f(z)=1+\sum_{n=1}^{\infty} a_{n} z^{n}$ for $|z|<\rho$, then since $f(z)$ is univalent it follows that the function $(f(z)-1) / a_{1}$ is a member of $T M(\rho)$. We thus obtain from (2.1) the inequality,

$$
\left|a_{n}\right| \leqslant \frac{1-\rho^{2 n}}{\rho^{n-1}\left(1-\rho^{2}\right)}\left|a_{1}\right|
$$

for all $n$. In [8], it was proven that $\left|a_{1}\right| \leqslant(1+\rho)^{2} / \rho$. Combining this with (2.2) we obtain

$$
\left|a_{n}\right| \leqslant\left(\frac{1+\rho}{1-\rho}\right)\left(\frac{1-\rho^{2 n}}{\rho^{n}}\right)
$$

for all $n$. The interesting thing here however, is that under the assumption that $f(z)$ is real for real values of $z$, the coefficients $a_{n}$ are necessarily positive and we can obtain precise lower bounds.

THEOREM 1. If $f(z)$ is in $\Lambda^{*}(\rho)$ and real for real values of $z$ and if $f(z)=1+\sum_{n=1}^{\infty} a_{n} z^{n}$ for $|z|<\rho$, then for all $n$

$$
\left(\frac{1-\rho}{1+\rho}\right)\left(\frac{1-\rho^{2 n}}{\rho^{n}}\right) \leqslant a_{n} \leqslant\left(\frac{1+\rho}{1-\rho}\right)\left(\frac{1-\rho^{2 n}}{\rho^{n}}\right) .
$$

The inequalities are sharp for each $n$. Equality is attained on the right side of (2.4) for each $n$ by the function $f(z)=-\rho(1+z)^{2} /(z-\rho)(1-\rho z)$ and on the left side of (2.4) for each $n$ by the function $f(z)=-\rho(1-z)^{2} /(z-\rho)(1-\rho z)$.

REMARK. It was proven in [8] that the function

$$
f(z)=-\rho(1-z)^{2} /(z-\rho)(1-\rho z)
$$

is actually in $\Lambda(\rho)$ for all $\rho$ whereas the function

$$
f(z)=-\rho(1+z)^{2} /(z-\rho)(1-\rho z)
$$

is in $\Lambda(\rho)$ for $\rho \leqslant 1 / 2$ but is in $\Lambda^{*}(\rho) \backslash \Lambda(\rho)$ for $\rho>1 / 2$. Thus the left-hand 
side of (2.4) is sharp in $\Lambda(\rho)$ for all $\rho$ and the right side is sharp in $\Lambda(\rho)$ at least for $\rho<1 / 2$.

Proof OF TheOREM 1. It follows from (1.3) that the funtion

$$
P(z)=z f^{\prime}(z) / f(z)+\rho /(z-\rho)-\rho z /(1-\rho z) .
$$

has negative real part in $\Delta$. Let

$$
\frac{z f^{\prime}(z)}{f(z)}=\sum_{n=1}^{\infty} b_{n} z^{n}
$$

for $|z|<\rho$. Then from (2.5) we obtain

$$
P(z)=-1+\sum_{n=1}^{\infty}\left(b_{n}-\rho^{n}-\frac{1}{\rho^{n}}\right) .
$$

Since $P(z)$ has negative real part in $\Delta$ it follows that for each $n$

$$
\left|b_{n}-\rho^{n}-1 / \rho^{n}\right| \leqslant 2
$$

Since by assumption $f(z)$ is real for real values of $z$, the coefficients $b_{n}$ are real and we have from (2.7)

$$
\left(1-\rho^{n}\right)^{2} / \rho^{n} \leqslant b_{n} \leqslant\left(1+\rho^{n}\right)^{2} / \rho^{n}
$$

for all $n$. From (2.6) we have that

$$
z f^{\prime}(z)=\left(\sum_{n=1}^{\infty} b_{n} z^{n}\right) f(z)
$$

for $|z|<\rho$. Comparing the coefficients of both sides of (2.9) we obtain

$$
n a_{n}=b_{n}+a_{1} b_{n-1}+a_{2} b_{n-2}+\cdots+a_{n-1} b_{1}
$$

for all $n$. Thus in particular $a_{1}=b_{1} \geqslant(1-\rho)^{2} / \rho$ which is the left side of (2.4) for $n=1$. Next suppose that $a_{k} \geqslant((1-\rho) /(1+\rho))\left((1-\rho)^{2 k} / \rho^{k}\right)$ for $k=1,2, \ldots,(n-1)$. Then from (2.4) we obtain

$$
\begin{aligned}
n a_{n} & \geqslant \frac{\left(1-\rho^{n}\right)^{2}}{\rho^{n}}+\sum_{k=1}^{n-1} \frac{(1-\rho)}{(1+\rho)} \frac{\left(1-\rho^{2 k}\right)\left(1-\rho^{n-k}\right)^{2}}{\rho^{n}} \\
& =\frac{(1-\rho)}{\rho^{n}(1+\rho)}\left[\left(1-\rho^{2}\right)\left(\sum_{k=0}^{n-1} \rho^{k}\right)^{2}+\sum_{k=1}^{n-1}\left(1-\rho^{2 k}\right)\left(1-\rho^{n-k}\right)^{2}\right] \\
& =\frac{(1-\rho)}{\rho^{n}(1+\rho)}\left[( 1 - \rho ^ { 2 } ) \left(1+2 \rho+3 \rho^{2}+\cdots+n \rho^{n-1}+(n-1) \rho^{n}\right.\right. \\
& \left.+(n-2) \rho^{n+1}+\cdots \rho^{2 n-2}\right)+(n-1)\left(1-\rho^{2 n}\right) \\
& =n((1-\rho) /(1+\rho))\left(\left(1-\rho^{2 n}\right) / \rho^{n}\right) .
\end{aligned}
$$


Thus the left side of (2.4) follows for all $n$. Due to the remarks preceding Theorem 1, the right side of (2.4) now follows. In conclusion, we remark that the right side of (2.4) cannot be obtained for all $n$ by making use of $(2.10)$ and the right side of (2.8).

CoROllary 1. Let $f(z)$ be in $\Lambda^{*}(\rho)$. If $f(z)=1+\sum_{n=1}^{\infty} a_{n} z^{n}$ for $|z|<\rho$ and if $a_{n}$ is real for $1 \leqslant n \leqslant N$, then

$$
a_{n} \geqslant((1-\rho) /(1+\rho))\left(\left(1-\rho^{2 n}\right) / \rho^{n}\right)
$$

for $1 \leqslant n \leqslant N$. The inequalities are sharp.

Proof. If $z f^{\prime}(z) / f(z)=P(z)=\sum_{n=1}^{\infty} b_{n} z^{n}$ for $|z|<\rho$, then the assumptions of the corollary imply that $b_{n}$ is real for $1 \leqslant n \leqslant N$. Thus (2.8) holds for $1 \leqslant n \leqslant N$. With this observation the proof proceeds as in Theorem 1 .

CoROllary 2. Let $f(z)$ be in $\Lambda^{*}(\rho)$. If $f(z)=1+\sum_{n=1}^{\infty} a_{n} z^{n}$ for $|z|<\rho$ and if $a_{n}$ is real for $1 \leqslant n \leqslant N$ then

$$
\operatorname{Re} a_{N+1} \geqslant((1-\rho) /(1+\rho))\left(\left(1-\rho^{2 N+1}\right) / \rho^{N+1}\right) .
$$

Proof. As in the proof of Corollary 1 the coefficients $b_{n}$ are real for $1 \leqslant n \leqslant N$. Thus taking real parts of both sides of (2.10) when $n=N+1$, we can follow the argument in the proof of Theorem 1 to obtain the desired inequality.

In [3] Goodman conjectured that if $f(z)$ is meromorphic and univalent in $\Delta$ with a simple pole at $z=p, 0<\rho<1$, and if $f(z)=z+\sum_{n=2}^{\infty} b_{n} z_{n}$ for $|z|<\rho$ then $\left|b_{n}\right| \leqslant\left(1-\rho^{2 n}\right) / \rho^{n-1}\left(1-\rho^{2}\right)$. Subsequently Jenkins [7] proved that if the Bieberbach conjecture is valid up to index $N$, then Goodman's conjecture is also valid up to index $N$. Using the fact that if $f(z)=1+$ $\sum_{n=1}^{\infty} a_{n} z^{n}$ is a member of $\Lambda^{*}(\rho),(f(z)-1) / a_{1}$ is univalent in $\Delta$ with a simple pole at $z=\rho$ and using the fact that at $\left|a_{1}\right| \leqslant\left(1+\rho^{2}\right) / \rho$ [8], it follows that

$$
\left|a_{n}\right| \leqslant((1+\rho) /(1-\rho))\left(\left(1-\rho^{2 n}\right) / \rho^{n}\right), \quad n \leqslant 6 .
$$

With the assumption that $f(z)$ in $\Lambda^{*}(\rho)$ is real on the real axis we have obtained positive lower bounds on $a_{n}$ for all $n$. It is natural then to ask whether there is in general a positive lower bound on $\left|a_{n}\right|$ for all $f(z)$ in $\Lambda^{*}(\rho)$.

3. Integral means. In this section we prove that the function

$$
F(z)=-\rho(1+z)^{2} /(z-\rho)(1-\rho z)
$$

has maximal $L^{p}$ means in the class $\Lambda^{*}(\rho)$.

Theorem 5. Let $f(z)$ be in $\Lambda^{*}(\rho)$ and $F(z)=-\rho(1+z)^{2} /(z-\rho)(1-\rho z)$ then for any positive real number $p$ and $r \neq \rho$,

$$
\int_{0}^{2 \pi}\left|f\left(r e^{i \theta}\right)\right|^{p} d \theta \leqslant \int_{0}^{2 \pi}\left|F\left(r e^{i \theta}\right)\right|^{p} d \theta .
$$

Proof. Since $f(z)$ is a member of $\Lambda^{*}(\rho)$ we can write

$$
f(z)=-\rho z G(z) /(z-\rho)(1-\rho z)
$$


where $G(z)$ is in $\Sigma^{*}$. Since $G(z)$ is in $\Sigma^{*}$ there exists a nondecreasing function $m(t)$ on $[-\pi, \pi]$ with $\int_{-\pi}^{\pi} d m(t)=1$ such that

$$
G(z)=\frac{1}{z} \exp \left(2 \int_{-\pi}^{\pi} \log \left(1-e^{-i t} z\right) d m(t)\right) .
$$

Thus from (3.2) and (3.3) we have

$$
f(z)=\frac{-\rho}{(z-\rho)(1-\rho z)} \exp \left(2 \int_{-\pi}^{\pi} \log \left(1-e^{-i t} z\right) d m(t)\right)
$$

Using the continuous form of the arithmetic-geometric mean inequality $[9, \mathrm{p}$. 64] we obtain for $r \neq \rho$ and $p>0$

$$
\begin{aligned}
\left|f\left(r e^{i \theta}\right)\right|^{p} & =\frac{\rho^{p}}{\left|r e^{i \theta}-\rho\right|^{p}\left|1-\rho r e^{i \theta}\right|^{p}} \exp \left(\int_{-\pi}^{\pi} \log \left|1-r e^{i(\theta-t)}\right|^{2 p} d m(t)\right) \\
& \leqslant \frac{\rho^{p}}{\left|r e^{i \theta}-\rho\right|^{p}\left|1-\rho r e^{i \theta}\right|^{p}}\left(\int_{-\pi}^{\pi}\left|1-r e^{i(\theta-t)}\right|^{2 p} d m(t)\right) .
\end{aligned}
$$

From (3.5) we thus have

$$
\begin{aligned}
\int_{0}^{2 \pi}\left|f\left(r e^{i \theta}\right)\right|^{p} d \theta & \leqslant \int_{-\pi}^{\pi}\left[\int_{-\pi}^{\pi} \frac{\rho^{p}\left|1-r e^{i(\theta-t)}\right|^{2 p}}{\left|r e^{i \theta}-\rho\right|^{p}\left|1-\rho r e^{i \theta}\right|^{p}} d m(t)\right] d \theta \\
& =\int_{-\pi}^{\pi}\left[\int_{-\pi}^{\pi} \frac{\rho^{p} \mid 1-r e^{\left.i(\theta-t)\right|^{2 p}}}{\left|r e^{i \theta}-\rho\right|^{p}\left|1-\rho r e^{i \theta}\right|^{p}} d \theta\right] d m(t) \\
& \left.\leqslant \int_{-\pi}^{\pi}\left[\max _{-\pi<t<\pi} \int_{-\pi}^{\pi} \frac{\rho^{p} \mid 1-r e^{\left.i(\theta-t)\right|^{2 p}}}{\left|r e^{i \theta}-\rho\right|^{p}\left|1-\rho r e^{i \theta}\right|^{p}} d \theta\right] d m(t)\right] \\
& =\rho^{p} \max _{-\pi<t<\pi} \int_{-\pi}^{\pi} \frac{\left|1-r e^{i(\theta-t)}\right|^{2 p}}{\left|r e^{i \theta}-\rho\right|^{p}\left|1-\rho r e^{i \theta}\right|^{p}} d \theta .
\end{aligned}
$$

Let

$$
I(t)=\int_{-\pi}^{\pi} \frac{\left|1-r e^{i(\theta-t)}\right|^{2 p}}{\left|r e^{i \theta}-\rho\right|^{p}\left|1-\rho r e^{i \theta}\right|^{p}} d \theta .
$$

According to (3.6), the theorem will be proven if it can be shown that

$$
I(t) \leqslant \int_{-\pi}^{\pi} \frac{\left|1+r e^{i \theta}\right|^{2 p}}{\left|r e^{i \theta}-\rho\right|^{p}\left|1-\rho r e^{i \theta}\right|^{p}} d \theta
$$

for $-\pi \leqslant t \leqslant \pi$. However, Clunie and Duren [2] have proven the following: Given a nonnegative measurable function $F(x)$ on $[-a, a]$, let $F^{*}(x)$ denote its symmetrically decreasing rearrangement as defined in [4, p. 278]. If $F(x)$, 
$G(x)$ and $H(x)$ are nonnegative integrable functions on the interval [ $-a, a]$, then

$$
\int_{-a}^{a} F(x) G(x) H(x) d x \leqslant \int_{-a}^{a} F^{*}(x) G^{*}(x) H^{*}(x) d x .
$$

Since for any $t$ the rearrangement of $\left|1-r e^{i(\theta-t)}\right|^{2 p}$ is $\left|1+r e^{i \theta}\right|^{2 p}$ and since $\left|\rho-r e^{i \theta}\right|^{-p}$ and $\left|1-\rho r e^{i \theta}\right|^{-p}$ are equal to their rearrangements, an application of (3.9) to (3.7) gives (3.8) and hence the theorem.

Corollary. Let $f(z)$ be in $\Lambda^{*}(\rho)$. If

$$
f(z)=\sum_{n=1}^{\infty} B_{-n} z^{-n}+\sum_{n=0}^{\infty} B_{n} z^{n} \text { for } \rho<|z|<1,
$$

then

$$
\sum_{n=1}^{\infty}\left|B_{-n}\right|^{2}+\sum_{n=0}^{\infty}\left|B_{n}\right|^{2} \leqslant \frac{2 \rho^{2}}{(1-\rho)^{2}}\left[\frac{1+\rho}{1-\rho}+2\right]
$$

Proof. From Theorem 5 we have that for $\rho<r<1$

$$
\frac{1}{2 \pi} \int_{0}^{2 \pi}\left|f\left(r e^{i \theta}\right)\right|^{2} d \theta \leqslant \frac{1}{2 \pi} \int_{0}^{2 \pi}\left|F\left(r e^{i \theta}\right)\right|^{2} d \theta
$$

where $F(z)=-\rho(1+z)^{2} /(z-\rho)(1-\rho z)$. If we let $F(z)=\sum_{n=1}^{\infty} A_{-n} z^{-n}+$ $\sum_{n=0}^{\infty} A_{n} z^{n}$ for $\rho<|z|<1$ then (3.11) implies that

$$
\sum_{n=1}^{\infty}\left|B_{-n}\right|^{2} r^{-2 n}+\sum_{n=0}^{\infty}\left|B_{n}\right|^{2} r^{2 n} \leqslant \sum_{n=1}^{\infty}\left|A_{-n}\right|^{2} r^{-2 n}+\sum_{n=0}^{\infty}\left|A_{n}\right|^{2} r^{2 n}
$$

But it is easily seen that $A_{-n}=A_{n}=-\rho^{n}(1+\rho) /(1-\rho)$ for $n=1,2$, $3, \ldots$ and $A_{0}=-2 \rho /(1-\rho)$. Using these values in (3.12) we obtain

$$
\begin{aligned}
& \sum_{n=1}^{\infty}\left|B_{-n}\right|^{2} r^{-2 n}+\sum_{n=0}^{\infty}\left|B_{n}\right|^{2} r^{2 n} \\
& \leqslant\left(\frac{1+\rho}{1-\rho}\right)^{2}\left[\frac{\rho^{2}}{r^{2}-\rho^{2}}+\frac{\rho^{2} r^{2}}{1-\rho^{2} r^{2}}\right]+\frac{4 \rho^{2}}{(1-\rho)^{2}} .
\end{aligned}
$$

Letting $r$ approach 1 in (3.13) gives (3.10).

\section{REFERENCES}

1. J. Clunie, On meromorphic schlicht functions, J. London Math. Soc. 34 (1959), 215-216. MR 21 \# 5737.

2. J. Clunie and P. L. Duren, An arclength problem for close-to-convex functions, J. London Math. Soc. 39 (1964), 757-761; Addendum, ibid. 41 (1966), 181-182. MR 30 \#241; 32 \#725.

3. A. W. Goodman, Functions typically real and meromorphic in the unit circle, Trans. Amer. Math. Soc. 81 (1956), 92-105. MR 17, 724.

4. G. H. Hardy, J. E. Littlewood and G. Pólya, Inequalities, Cambridge Univ. Press, New York, 1959. (For review of 2nd ed., 1952, see MR 13, 727.) 
5. J. Hummel, Extremal properties of weakly starlike p-valent functions, Trans. Amer. Math. Soc. 130 (1968), 544-551. MR 36 \#5332.

6., The coefficients of starlike functions, Proc. Amer. Math. Soc. 22 (1969), 311-315. MR $40 \# 4440$.

7. J. A. Jenkins, On a conjecture of Goodman concerning meromorphic univalent functions, Michigan Math. J. 9 (1962), 25-27. MR 24 \# A2017.

8. R. J. Libera and A. E. Livingston, Weakly starlike meromorphic univalent functions, Trans. Amer. Math. Soc. 202 (1975), 181-191. MR 50 \# 13482.

9. W. Rudin, Real and complex analysis, 2nd ed., McGraw-Hill, New York, 1974.

Department of Mathematics, University of Delaware, Newark, Delaware 19711 